\title{
Immunogold study of non-collagenous matrix components in normal and exfoliative iris
}

\author{
A Vogiatzis, G E Marshall, A G P Konstas, W R Lee
}

\begin{abstract}
The present investigation was undertaken to determine if some of the components of exfoliation material in iris tissue were unique to exfoliation or were part of normal iris architecture. Eleven normal iris specimens and $\mathbf{1 0}$ exfoliative iris specimens were processed for cryoultramicrotomy and London resin white embedding. Immunogold electron microscopy was used to investigate the fine structural distribution of amyloid P component, elastin, entactin, fibronectin, gp115, and vitronectin in normal iris and their association with exfoliation material. Exfoliation material was positive for amyloid $\mathrm{P}$ component and possibly gp115, neither of which were present in normal iris tissue. Elastin and fibronectin were present in the normal iris stroma but were not associated with exfoliation material. The distribution of amyloid $\mathbf{P}$ component in the vessel lumen and wall led to the conclusion that amyloid $\mathbf{P}$ is a serum contaminant. The presence of gp115 in exfoliation material represents the synthesis of a component novel to the iris vascular cell synthetic repertoire.
\end{abstract}

(Br f Ophthalmol 1994; 78: 850-858)

The exfoliation syndrome or pseudoexfoliation syndrome is considered to be a disorder in which there is an abnormal production and deposition of extracellular matrix in intra- and extraocular tissues. ${ }^{1-3}$ Exfoliation syndrome is an age-related disease which is often complicated by glaucoma. ${ }^{45}$

By light microscopy exfoliation material is seen

Tennent Institute of Ophthalmology, Glasgow A Vogiatzis

W R Lee

Department of

Pathology, Western

Infirmary, University of

Glasgow, Glasgow

GE Marshall

W R Lee

University Department of Ophthalmology, AHEPA Hospital,

Thessaloniki, Greece

A G P Konstas

Correspondence to:

G E Marshall, Tennent

Institute of Ophthalmology,

Western Infirmary,

Wniversity of Glasgow,

Glasgow G11 6NT.

Accepted for publication

15 June 1994 as eosinophilic bush-shaped accretions attached to zonular fibres, the surfaces of the ciliary processes, lens and iris, and within the trabecular meshwork..$^{6-8}$ By transmission electron microscopy, exfoliation material appears as a polydimensional accumulation of cotton-like fibres consisting of filamentous subunits, with a varied periodicity $(17-56 \mathrm{~nm})$ embedded in an interfibrillar matrix. ${ }^{2}$ The biochemical nature of exfoliation material has been the subject of intensive study but the exact composition remains to be elucidated. It is not known which of the constituents are the primary products of disordered cell secretion or which are simply absorbed into the abnormal matrix.

Several extracellular matrix (ECM) components have been localised to exfoliation material using the immunogold technique on post- embedded tissue, including amyloid $\mathrm{P}$ component, ${ }^{9-11}$ chondroitin sulphate, ${ }^{910}$ elastin, ${ }^{911}$ entactin (nidogen), ${ }^{10}$ fibronectin, ${ }^{10}$ heparan sulphate, ${ }^{910}$ laminin, ${ }^{101213}$ and vitronectin. ${ }^{9}$

The post-embedding immunolabelling technique carries the risk of masking antigenic epitopes. It is therefore more desirable to apply the cryoultramicrotomy technique in which immunolabelling is conducted before embedding, thus allowing a greater access to antigenic epitopes. It is, however, important that both cryoultramicrotomy and plastic embedding techniques are applied when it is necessary to identify the constituents of an abnormal accumulation of a complex biochemical nature.

In terms of pathogenesis, the accumulation of exfoliation material may simply be due to an uncontrolled production of ECM components by cells which are normally engaged in their synthesis. Alternatively, the cells may be triggered to synthesise ECM components completely outside their normal repertoire. Thus it is necessary to determine the status of these components in normal tissue. We therefore studied the distribution of a select number of ECM components namely, amyloid P component, elastin, entactin, fibronectin, and vitronectin, in the normal iris and to confirm their presence in exfoliation material. To further investigate the elastic constituents, we also used a recently commercially available antibody to elastin associated glycoprotein (gpl15).

\section{Materials and methods}

Normal iris tissue was obtained from four surgical enucleations, two postmortem enucleations (within 1.5 and 3 hours of death respectively) and six iridectomies (see Table 1 for clinical details). All specimens were considered normal by histological examination of toluidine blue stained semithin $(1 \mu \mathrm{m})$ London resin (LR) white plastic embedded sections. Ten exfoliative iridectomy specimens were obtained from patients operated on for exfoliation glaucoma (Table 2).

It is well recognised that the level of glutaraldehyde concentration can have profound effects on the degree of antigenic preservation: for this reason specimens were exposed to concentrations of glutaraldehyde varying from $0 \%$ to $1 \%$ (see Tables 1 and 2 ).

\section{FIXATION}

Normal iris tissue obtained from surgical and 
Table 1 Patient and processing details of normal iris specimens

\begin{tabular}{lllllll}
\hline Case & Age & Sex & Diagnosis & Operation & Technique & $\begin{array}{l}\text { Fixation } \\
(\% \text { glutaraldehyde } \\
+4 \% \text { PFA })\end{array}$ \\
\hline 1 & 35 & F & Orb tumour & E & Cryo & PFA only \\
2 & 38 & F & CM & E & Cryo & $0 \cdot 5 \%$ \\
3 & 40 & M & PM & E & Cryo & $0 \cdot 5 \%$ \\
4 & 52 & M & CM & E & LRW & $0 \cdot 1 \%$ \\
5 & 63 & M & & I & LRW & PFA only \\
6 & 64 & F & & I & LRW & PFA only \\
7 & 67 & F & PM & I & LRW & PFA only \\
8 & 80 & F & Pec glaucoma & E & Cryo & $0 \cdot 75 \%$ \\
9 & 81 & F & LRW & $0 \cdot 1 \%$ \\
10 & 85 & M & & I & LRW & PFA only \\
11 & 93 & F & & I & LRW & PFA only \\
\hline
\end{tabular}

$\mathrm{CM}=$ choroidal melanoma. $\mathrm{PM}=$ post mortem $. \mathrm{E}=$ enucleation. $\mathrm{I}=$ iridectomy.

Cryo= cryoultramicrotomy. $\mathrm{LRW}=\mathrm{LR}$ white.

Table 2 Patient and processing details of exfoliative iris specimens

\begin{tabular}{lllllll}
\hline Case & Age & Sex & Diagnosis & Operation & Technique & $\begin{array}{c}\text { Fixation } \\
(\% \text { glutaraldehyde } \\
+4 \% \text { PFA) }\end{array}$ \\
\hline 1 & 62 & M & EXF glaucoma & I & LRW & $0 \cdot 1 \%$ \\
2 & 68 & M & EXF glaucoma & I & LRW & $0 \cdot 1 \%$ \\
3 & 68 & F & EXF syndrome & I & LRW & $0 \cdot 1 \%$ \\
4 & 71 & M & EXF syndrome & I & LRW & $0 \cdot 1 \%$ \\
5 & 72 & F & EXF syndrome & I & LRW & $0 \cdot 1 \%$ \\
6 & 78 & F & EXF syndrome & I & LRW & $0 \cdot 1 \%$ \\
7 & 82 & M & EXF glaucoma & I & LRW & PFA only \\
8 & & M & EXF glaucoma & I & LRW & $0 \cdot 4 \%$ \\
9 & & & & & Cryo & \\
10 & & & & & Cryo & \\
\hline
\end{tabular}

EXF $=$ exfoliation. $\mathrm{I}=$ iridectomy. Cryo $=$ cryoultramicrotomy. $\mathrm{LRW}=\mathrm{LR}$ white.

Table 3 Details of primary antibodies

\begin{tabular}{|c|c|c|c|c|c|}
\hline \multirow[b]{2}{*}{ Antigen } & \multirow[b]{2}{*}{ Type } & \multicolumn{2}{|c|}{ Dilution } & \multirow[b]{2}{*}{ Company } & \multirow[b]{2}{*}{ Specificity tests } \\
\hline & & $\overline{L R W}$ & Cryo & & \\
\hline Amyloid P & Rabbit poly & $1: 140$ & $1: 1200$ & Dako, Denmark & $\begin{array}{l}\text { Crossed and rocket } \\
\text { immunoelectrophoresis } \\
\text { Single radial } \\
\text { immunodiffusion }\end{array}$ \\
\hline Elastin & Mouse mono & $1: 100$ & $1: 100$ & Sigma, UK & $\begin{array}{l}\text { Immunoaffinity } \\
\text { chromatography }\end{array}$ \\
\hline Entactin & Mouse poly & $1: 100$ & $1: 50$ & UBI, USA & Western blot \\
\hline Fibronectin & Mouse mono & $1: 100$ & $1: 50$ & Sigma, UK & Immunoblot \\
\hline gpll15 & Mouse mono & $1: 150$ & $1: 800$ & Chemicon, USA & $\begin{array}{l}\text { Immunoblotting } \\
\text { Immunoprecipitation }\end{array}$ \\
\hline Vitronectin & Mouse mono & $1: 100$ & $1: 50$ & $\begin{array}{l}\text { Manheim } \\
\text { Boehringer, } \\
\text { Germany }\end{array}$ & $\begin{array}{l}\text { ELISA, } \\
\text { Western blot }\end{array}$ \\
\hline
\end{tabular}

postmortem enucleations was used as control material. This material had been fixed in $4 \%$ paraformaldehyde (PFA) and $0 \cdot 1-1 \cdot 0 \%$ glutaraldehyde in $0.1 \mathrm{M}$ cacodylate or phosphate buffer $(\mathrm{pH} \mathrm{7 \cdot 4)}$ at room temperature for 2 hours and immediately processed (see below). In the current study, control iridectomies from primary open angle glaucoma cases were fixed in 4\% PFA (cacodylate or phosphate buffer $\mathrm{pH} 7 \cdot 4$ ) for 1.5-2 hours at room temperature. Six of the exfoliative iridectomies were fixed in $0.1 \%$ glutaraldehyde (cacodylate buffer) for 1.5 hours and the other four exfoliative iridectomies in $4 \%$ PFA ( 1.5 hours). Blocks were washed three times in buffer ( 5 minutes each) and allocated for cryoultramicrotomy and LR white embedding. LR white is a hydrophilic hydroxymethacrylate resin which can be used to embed tissue that has been dehydrated in $70 \%$ alcohol, thereby allowing greater preservation of antigenicity. ${ }^{14}$

LR WHITE PLASTIC EMBEDDING

Blocks allocated for LR white plastic embedding were dehydrated by 15 minute changes in $20 \%$ and $50 \%$ ethanol, block stained with $2 \%$ uranyl acetate in $70 \%$ ethanol for 1 hour, washed in three 20 minute changes of $70 \%$ ethanol, and infiltrated by two, 1 hour changes of LR white resin (hard grade). The above steps were carried out at $4^{\circ} \mathrm{C}$. Tissue blocks were embedded in $\mathrm{OO}$ size sealed gelatin capsules and the resin polymerised at $43^{\circ} \mathrm{C}$ for 48 hours.

Gold or silver coloured ultrathin sections were cut on an LKB Ultrotome Nova (Cambridge Instruments), mounted on 200 mesh nickel grids, allowed to dry for 2 hours and rehydrated with distilled water. The sections were quenched of free aldehyde groups with $0.5 \mathrm{M}$ ammonium chloride in $50 \mathrm{mM}$ Tris buffer (pH 7.2) for 30 minutes and then washed by four, 2 minute changes in $50 \mathrm{mM}$ Tris buffer before overnight preincubation in $1 \%$ bovine serum albumin (BSA, essentially immunoglobulin free, Sigma) in Tris buffer at $4^{\circ} \mathrm{C}$.

\section{CRYOULTRAMICROTOMY}

Blocks allocated for cryoultramicrotomy were infused overnight with $2.3 \mathrm{M}$ sucrose at $4^{\circ} \mathrm{C}$, mounted on silver pins, snap frozen, and stored in liquid nitrogen. Ultrathin frozen sections were cut at $-83^{\circ} \mathrm{C}$ on a Reichert Jung FC4D cryoultramicrotome (Cambridge Instruments) and transferred on $2 \cdot 3 \mathrm{M}$ sucrose drops to 200 mesh carbon Formvar composite nickel grids and preincubated on $1 \%$ BSA in Tris buffer overnight at $4^{\circ} \mathrm{C}$. Free aldehyde groups were quenched by two 5 minute changes in $30 \mathrm{mM}$ glycine in Tris buffer before incubation in the primary antibody.

\section{IMMUNOCYTOCHEMISTRY}

The Tris buffer employed throughout the immunocytochemical procedure was $50 \mathrm{mM}$ Tris $\mathrm{pH} 7.2$ with the addition of $1.5 \mathrm{~g} / \mathrm{l}$ of sodium azide, $0.5 \mathrm{M} \mathrm{NaCl}^{15}$ and $0.05 \%$ Tween 20 (Sigma). Tween 20 was omitted from the buffer when labelling ultrathin frozen sections (cryoultramicrotomy). Primary antibody incubation was conducted for 2 hours at room temperature with the addition of $1 \%$ BSA to the Tris buffer. Unbound antibody was removed by six 5 minute changes in buffer and the grids incubated for 5 minutes on $1 \%$ BSA (in Tris) before labelling with a 1:50 dilution of $10 \mathrm{~nm}$ immunogold conjugate (Biocell Laboratories). Excess immunogold antibody was removed by six 5 minute changes in Tris buffer and the grids subsequently washed in two changes of distilled water. LR white ultrathin sections were stained with saturated aqueous uranyl acetate for 12-15 minutes and ultrathin frozen sections (cryoultramicrotomy) were embedded in a 10:1 mixture of 3\% polyvinyl alcohol and saturated aqueous uranyl acetate. Grids were then examined in a Jeol 1200 EXII transmission electron microscope at $80 \mathrm{kV}$.

\section{ANTIBODIES}

The optimal working dilution of each antibody was determined experimentally. Higher dilu- 


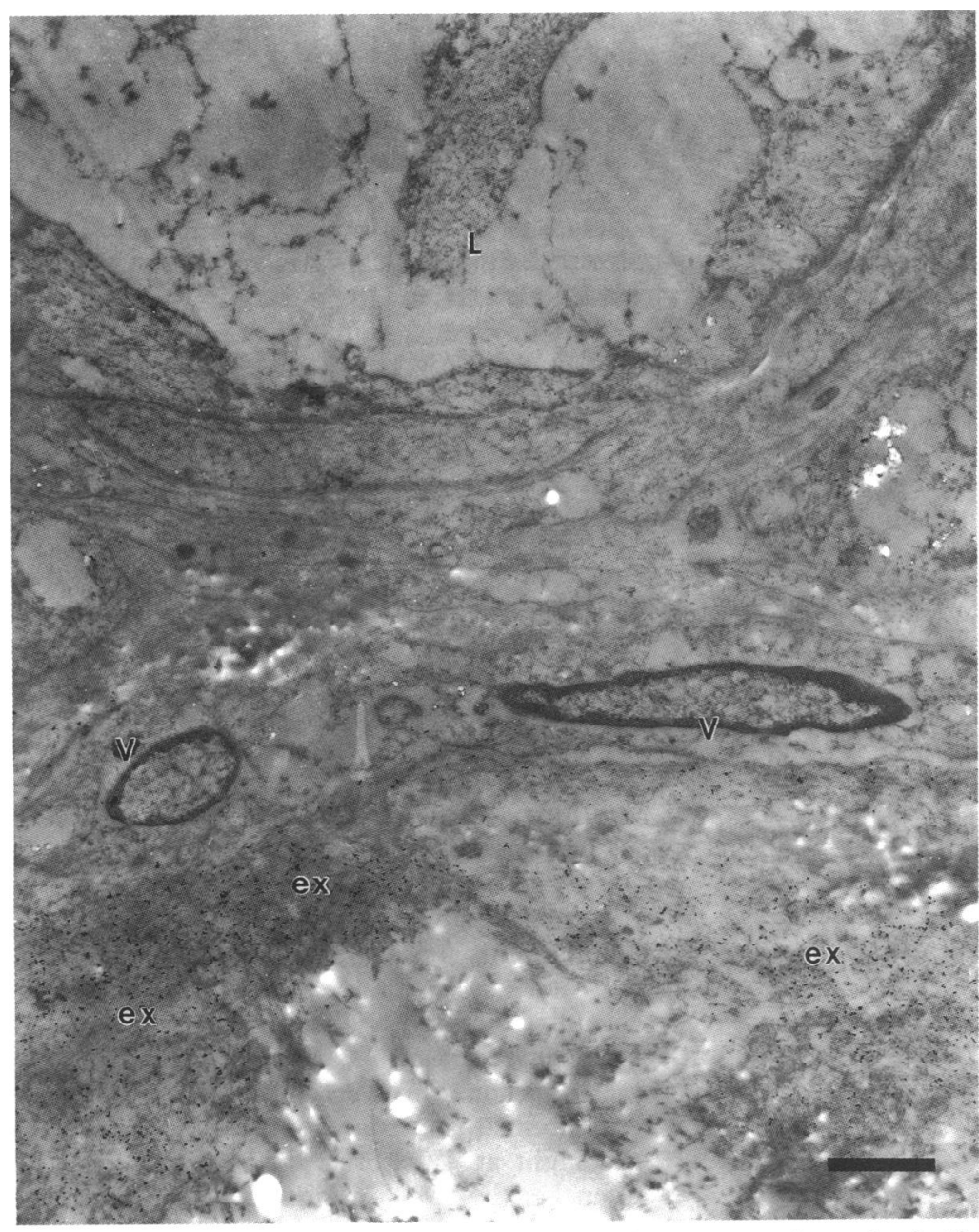

Figure $1 A$ Exfoliation aggregate (ex) labelled for amyloid $P$ component, is associated with vascular supporting cells $(V)$ of an exfoliative iris vessel. $(L)=$ vessel lumen. ( $L R$ white, $\times 11350$, bar $=1 \mu \mathrm{m})$.

Figure $1 B$ Higher power of Figure 1 . Immunogold labelling for amyloid $P$ component. Immunogold particles are localised to exfoliation material and are absent from electron lucent spaces within the aggregate, the cell nucleus $(\mathrm{Nu})$ and cytoplasm (Cyt). (LR white, $\times 18600$, $b a r=0.5 \mu \mathrm{m})$.

\section{CONTROLS}

Two negative controls were incorporated in the immunocytochemical procedure: (1) omission of the primary antibody, and (2) substitution of the primary antibody with non-immune serum from the same species in which the primary antibody was raised (Sigma). Non-immune sera dilutions were identical to those of the primary antibodies.

Immunogold labelling of the extracellular matrix was considered specific only when immunogold particles were absent from red blood cells, cell nuclei, mitochondria, and pigment granules. Non-specific labelling was best detected by examination of these structures.

\section{Results}

Exfoliative material was readily recognised in LR white embedded tissue but was not as easily differentiated from other structures in ultrathin frozen sections. Reliable identification required examination at low magnifications. Individual exfoliation fibres were thinner and more prominent in LR white embedded tissue, giving the impression of being shrunken or collapsed (Figs $1,2)$. In ultrathin frozen sections exfoliation fibres were often ooscured by the abundance of an associated matrix of similar electron density (Fig 3).

\section{AMYLOID P COMPONENT}

\section{Normal iris}

Amyloid P component was localised, in LR white embedded sections of normal iris, specifically to fine granular deposits of plasma within the lumen of blood vessels. Vessels which did not contain plasma were free of immunogold particles. Labelling intensity of the plasma did not vary with the type of fixative used. Antibodies against amyloid $\mathbf{P}$ component did not label basement membranes associated either with blood vessels, the posterior pigment epithelium, or the dilator and sphincter muscle. Labelling for amyloid $P$ component was absent from the stroma.

Amyloid P component was localised to the lumen of blood vessels in ultrathin frozen sections (cryoultramicrotomy) of normal iris. As in LR white embedded iris tissue, labelling was absent from basement membranes of blood vessels, the pigment epithelium, and sphincter muscle.

\section{Exfoliative iris}

Antibodies against amyloid $\mathbf{P}$ component specifically labelled exfoliation material both in LR white embedded tissue sections (Figs 1, 2) and in ultrathin frozen sections (Fig 3). More specifically, immunogold particles were present over both fibres and the granular matrix and labelling intensity for amyloid $\mathbf{P}$ component increased with increasing density of the exfoliation deposit. The degree of labelling was greater in ultrathin frozen sections than in LR white embedded tissue (compare Fig 3 with Figs 1, 2). Labelling of LR white embedded exfoliative fibres varied with the precise fixation regime used. The signal was strong when either $4 \%$ PFA 


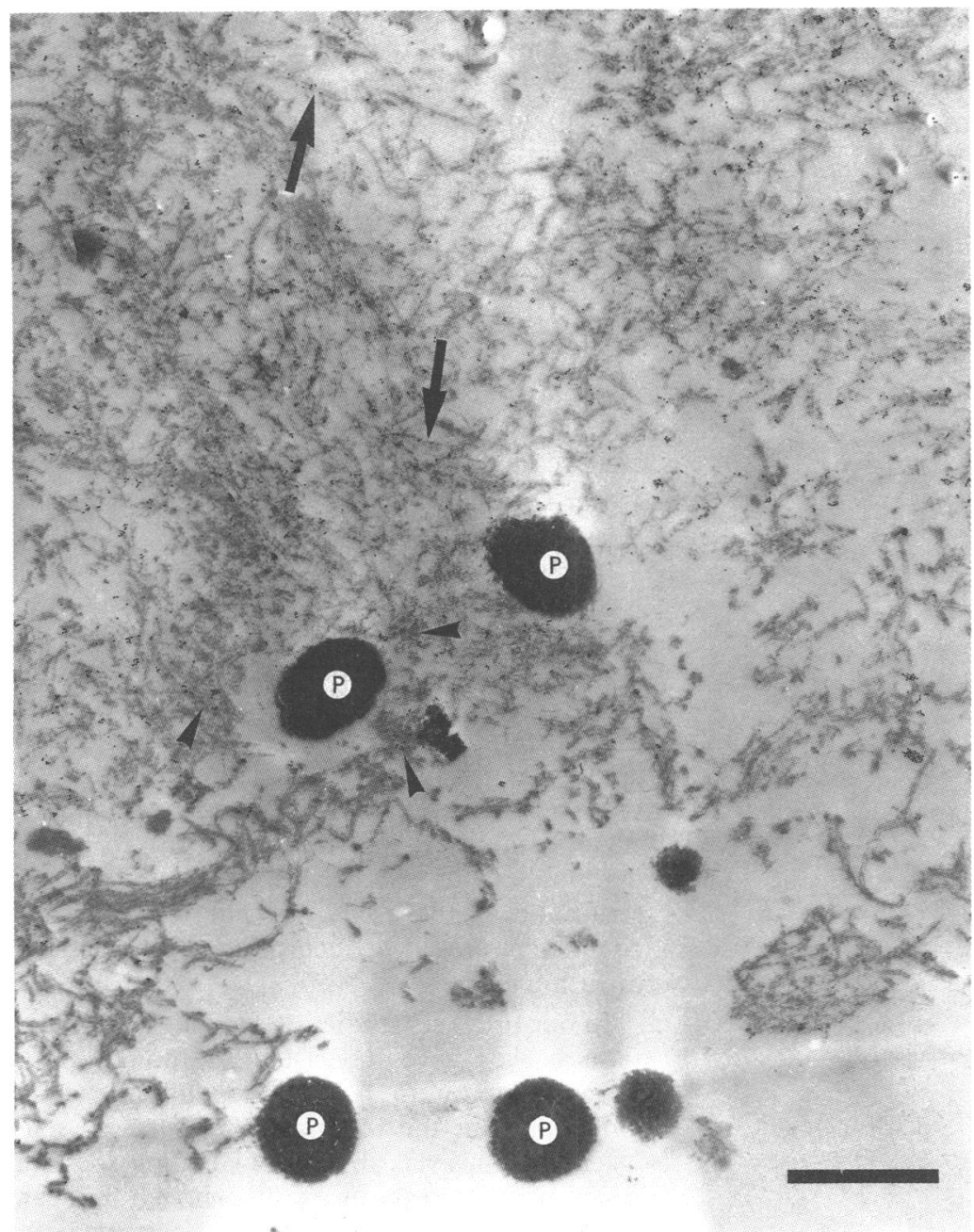

Figure 2 Exfoliation aggregate attached to posterior iris pigmen epithelium. Labelling for amyloid $P$ component is present both over exfoliative fibres (arrows) and the granular matrix (arrowheads). Pigment granules $(P)$ are present within the exfoliation deposit. (LR white, $\times 15500$, bar $=1 \mu \mathrm{m})$. or $0 \cdot 1 \%$ glutaraldehyde was employed and the use of paraglutaraldehyde (4\% PFA plus $0 \cdot 1-$ $0.5 \%$ glutaraldehyde), although significantly improving ultrastructural preservation, resulted in minimal and sometimes complete absence of labelling.

Immunogold labelling for amyloid $\mathbf{P}$ component was absent from areas free of exfoliation material in LR white embedded tissue. However, in ultrathin frozen sections, labelling was also present in the iris stroma at intensities comparable with those of the normal iris specimens. The precise distribution of immunogold particles was also similar, being present predominantly in the electron lucent regions of the stroma.

\section{ELASTIN}

Immunogold labelling for elastin was not observed in either normal or exfoliative iris embedded in LR white resin. Labelling for elastin was present in ultrathin frozen sections of both normal and exfoliation specimens. Immunogold particles were found in regions devoid of other identifiable matrix constituents (Fig 4) and occasionally the distribution of immunogold particles did not appear random but suggested the labelling of very thin filaments (Fig 4). Cellular elements, exfoliation fibres and their associated granular matrix were free of label as were areas of the carbon Formvar composite film from which tissue was absent.
ENTACTIN

Immunolabelling of both normal and exfoliative LR white embedded iris specimens failed to demonstrate the presence of entactin, regardless of the fixation regime employed. Similar negative results were obtained when labelling ultrathin frozen sections.

\section{FIBRONECTIN}

Fibronectin was not detected in any anatomical subcompartment in either normal or exfoliative iris tissue embedded in LR white plastic. However, some immunogold labelling was present in ultrathin frozen sections in both normal and exfoliative specimens to electron lucent regions in the iris stroma (Fig 5). Anti-fibronectin antibodies were not associated with either thick or thin collagen fibrils (Fig 5). Examination of the stroma at high magnification could not demonstrate the presence of fine filaments or any other discrete structural entities with which immunogold particles were associated. No labelling for fibronectin was observed over basement membranes. Cellular profiles and areas of the carbon Formvar composite film from which tissue was absent were devoid of immunogold particles. A similar pattern of labelling for fibronectin was also observed in ultrathin frozen sections of exfoliative iris tissue, but was absent from exfoliative deposits.

ELASTIN ASSOCIATED GLYCOPROTEIN (gp115)

Immunolabelling for elastic associated glycoprotein (gpl15) was absent from sections of normal iris prepared either by LR white embedding or by cryoultramicrotomy.

Labelling for gp115 was present in the stroma and over exfoliation deposits of exfoliative iris sections prepared by cryoultramicrotomy (Fig 6). Some association was observed between gp1 15 and striated collagen fibril bundles within the iris stroma. Immunogold particles were also randomly distributed over electron lucent areas of the stromal matrix and over aggregates of exfoliation material. The weak labelling of exfoliation deposits was in contrast with that observed for amyloid P component. Labelling observed in ultrathin frozen sections of exfoliative iris was not duplicated in sections of exfoliative specimens embedded in LR white plastic.

\section{VITRONECTIN}

Antibodies against vitronectin were not specifically localised to any of the anatomical subcompartments examined in either normal or exfoliative iris embedded in LR white plastic. Even light fixation (4\% PFA) did not permit positive identification of vitronectin. None of the exfoliation aggregates present in exfoliation specimens exhibited labelling for vitronectin. Increasing the concentration of primary antibody beyond a 1:50 dilution resulted in completely non-specific labelling of cells and extracellular matrix. The positive localisation of vitronectin was not achieved with ultrathin frozen sections of either normal or exfoliative iris. 


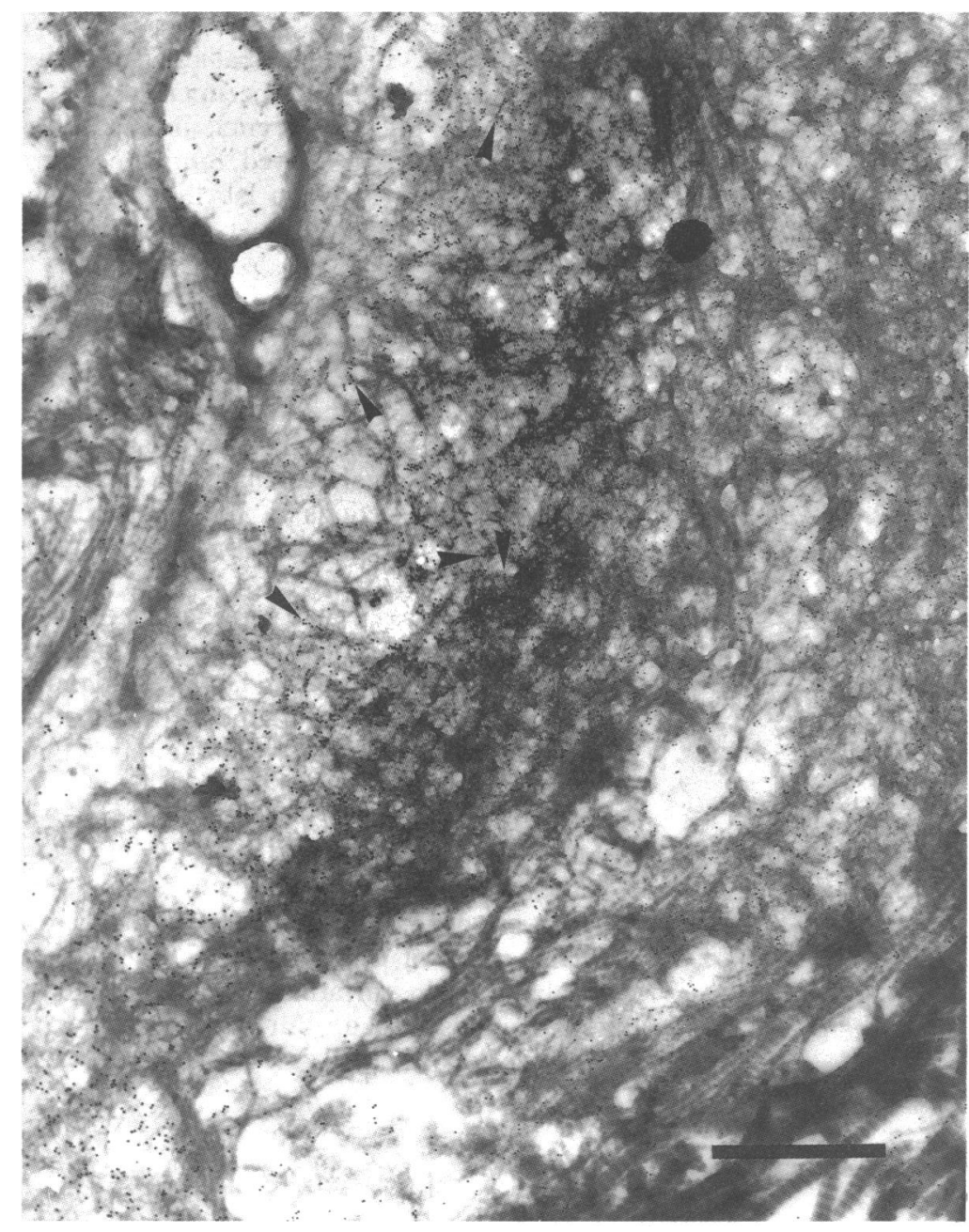

Figure 3 Ultrathin frozen section of exfoliation material labelled for amyloid $P$ component. Exfoliation fibres cannot be identified within the large amounts of amorphous matrix. Immunogold particles frequently lie in short rows (arrowheads) (cryo, $\times 18600$, bar $=$ $1 \mu \mathrm{m})$.
NEGATIVE CONTROLS

Immunogold particles were a rarity in rabbit anti-mouse and goat anti-rabbit immunogold negative control LR white or ultrathin frozen sections (omission of primary antibody). Immunogold labelling was not observed in the negative normal goat and rabbit serum control sections (substitution of the primary antibody with non-immune serum from the same species in which the primary antibody was raised).

\section{Discussion}

For more than seven decades a failure to identify the constituents of exfoliation material has led to controversies concerning its origin, nature, and pathogenesis. Streeten and co-workers ${ }^{1-316-18}$ postulated that exfoliation material is derived from abnormal polymerisation of glycoproteins associated with the zonular elastic microfibrillar system in a type of elastosis. This postulate is based on histochemical and immunohistochemical similarities between exfoliation material, the ocular zonules, ${ }^{18}{ }^{19}$ and elastic components. ${ }^{\prime}$

An alternative theory is that exfoliation material is 'an abnormal basement membrane synthesised at multiple sites by abnormal (aging) cells'.$^{20}$ This theory was based on the close proximity of exfoliation material with basement membranes, and the extensive basement membrane changes associated with exfoliative deposition. These changes are particularly prominent in basement membranes of the iris vasculature and a putative evolution of the vasculopathy has been constructed from extensive examination of iris blood vessels. ${ }^{21}$ Documentation of the distribution of collagen types $\mathrm{I}-\mathrm{V}$ and laminin in normal iris vessels ${ }^{122}$ provided a baseline for studying abnormalities in their distribution in exfoliative vasculopathy. Exfoliation deposits appeared to stimulate the deposition of collagen types I and IV (basement membrane collagen) by vascular supporting cells at an early stage of the disease, whereas advanced exfoliation vasculopathy was accompanied by a reduction in the amount of collagen types I and IV. ${ }^{13}$ An associated reduction in the laminin content of iris vessel walls was also noted. ${ }^{13}$

Considering the theories concerning the pathogenesis of the exfoliation syndrome it has been logical to attempt to identify components of the elastic system (amyloid P component, elastin and gp115) and the various glycoproteins which are known to be present in basement membranes (entactin and fibronectin). It is also reasonable to attempt to identify other components associated with the extracellular matrix such as vitronectin. A fundamental problem in this approach to determining the pathogenesis of the exfoliation syndrome is that some, if not all, of these components could be incorporated by a passive diffusion process.

In our previous studies of aged normal ocular tissues we have consistently found cryoultramicrotomy to be superior to LR white embedding in the localisation of type VI collagen. ${ }^{23-25}$ However, absence of labelling does not necessarily mean absence of the antigen, but can be attributed to failure of antigenic preservation, or lack of antibody specificity. The advantage of cryoultramicrotomy is that loss of antigenic preservation can only be due to fixation since subsequent steps in processing have no adverse effects on antigenicity.

An intrinsic disadvantage of the postembedding technique is that absence of labelling in LR white sections may result from inaccessibility of the antigenic epitope. LR white is a post-embedding technique in which only epitopes at the plastic section surface are available for antibody binding. ${ }^{14}$ Since embedding occurs after antibody labelling in the ultrathin frozen section technique, cryoultramicrotomy avoids this limitation. ${ }^{26}$

\section{ELASTOTIC COMPONENTS}

\section{Amyloid P component}

Using the two techniques of cryoultramicrotomy and LR white embedding we were able to confirm previous identification of amyloid $P$ component within exfoliation deposits. ${ }^{911}{ }^{17} \mathrm{How}$ ever, it cannot be inferred from this that exfoliation material is an amyloid-like substance, because amyloid $\mathrm{P}$ is only a minor component of primary amyloid, constituting about 5-10\% dry weight. ${ }^{27}$ The mandatory amyloid components (amyloid A, prealbumin, and immunoglobulin light chains) are absent from exfoliation substance."

Amyloid P component was localised, in the present study, to plasma within blood vessels of 


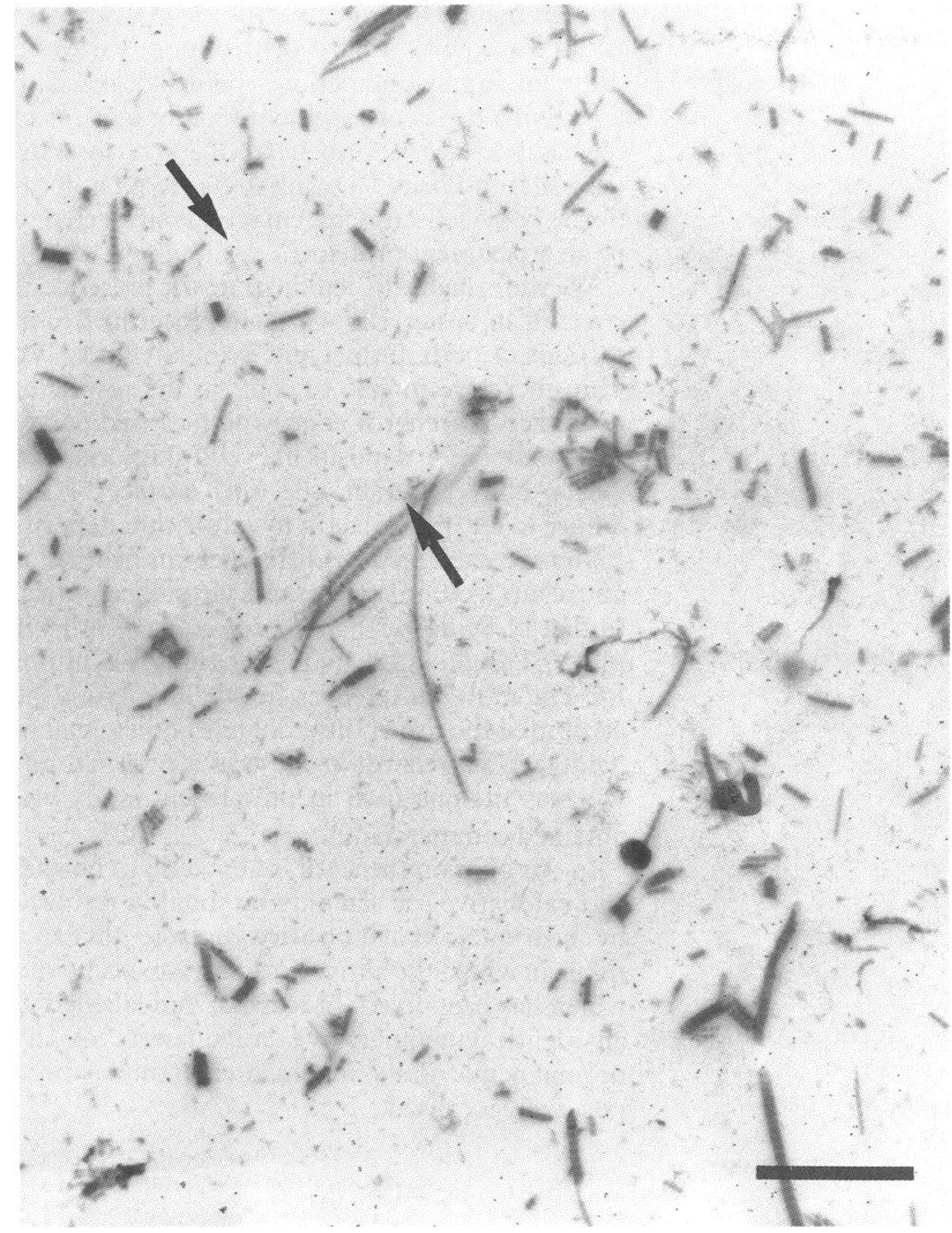

Figure 4 Elastin labelling in an ultrathin section of normal iris stroma reveals a linear distribution of immunogold particles (between arrows) suggesting the labelling of very fine filaments. No morphological detail of such putative filaments is evident (cryo, $\times 16750$, bar $=1 \mathrm{um}$. normal and exfoliative iris, but was absent from both vascular and epithelial basement membranes. Stromal labelling in control and exfoliative tissue may have arisen from selective binding of serum amyloid $P$ to some stromal component, probably fibronectin as seen by cryoultramicrotomy. De Beer et al ${ }^{28}$ demonstrated a calcium dependent selective binding of serum amyloid $P$ to fibronectin and C4-binding protein. The possibility of selective binding to fibronectin is substantiated by the fact that fibronectin was localised to the iris stroma but not to the vascular basement membranes which did not contain amyloid P. To date, amyloid P component has been shown to be an intrinsic component only of glomerular basement membranes and may be covalently linked to one of the normal basement membrane glycoproteins. ${ }^{29}$ Whether amyloid $\mathrm{P}$ is synthesised by the glomerular basement membrane producing cells or is simply serum amyloid $P$ that is incorporated into nascent basement membranes is unknown. ${ }^{29}$

We would concur with Li et al ${ }^{17}$ that amyloid P is not likely to be an intrinsic component of exfoliation material, but is the result of a ligand binding reaction, probably calcium dependent, of serum amyloid $P$. Although iris vessels maintain a blood-aqueous barrier, exfoliative iris vasculopathy is associated with increased vascular permeability. ${ }^{3011}$ This increase would facilitate leakage of serum amyloid $P$ component into the iris stroma and its subsequent binding to exfoliation material.

\section{Elastin}

The putative labelling of elastin in the normal stroma and the uninvolved stroma of exfoliative iris is, to the best of our knowledge, an original finding. The presence of small amounts of elastin in the iris stroma can be correlated with tissue function. Iris tissue undergoes both constant and rapid contraction and expansion with changes in pupil diameter and it would therefore be predictable that the iris should contain an elastic element. However, we wish to express reservations on the validity of our results, for although non-specific labelling over cellular elements was absent, the failure to associate elastin labelling with any visible structure is somewhat disconcerting.

With regard to elastin in exfoliation material, our results may initially seem contradictory to those previously published. Elastin and tropoelastin have been localised to the deposits by $\mathrm{Li}$ et al using two of three antibodies: labelling of the deposits with antibody BA-4 - a monoclonal antibody against bovine elastin - was considerably weaker. ${ }^{1}$ The same group ${ }^{11}$ found that exfoliation aggregates from a variety of organs (heart, lung, liver, gall bladder, and eye) stained positively for elastin. Schlotzer-Schrehardt et al, using the BA-4 monoclonal antibody, described moderate binding to exfoliation material in eyelid skin biopsies but only weak labelling of exfoliation material in the iris and ciliary body. In the present study using the BA-4 anti-bovine elastin monoclonal antibody we were unable to localise elastin to exfoliation substance in either LR white or ultrathin frozen sections. Using the same antibody we have successfully localised elastin to elastic fibres in the sclera (unpublished observations).

\section{Elastin associated glycoprotein (gp115)}

First isolated and characterised by Bressan et al ${ }^{32}$ it is not to be confused with a sialoglycoprotein of the same name (gpl15). ${ }^{33}$ The latter glycoprotein (also known as CD43, sialophorin, and leukosialin) is expressed on the surface of T lymphocytes, monocytes, neutrophils, platelets, and some B lymphocytes. ${ }^{3+}$ This confusion in nomenclature between two unrelated glycoproteins is the result of a historical artefact. Both glycoproteins were separated by sodium dodecyl sulphate polyacrylamide gel electrophoresis with an apparent molecular weight of 115000 and were termed gpl15 in different journals at the same time. . $^{33}$

Light microscopic immunohistochemistry of 2-day-old chicks has shown that elastin associated glycoprotein (gpl15) is present in connective tissue extracellular matrix of several organs, ${ }^{34}$ where it exhibits co-distribution with tropoelastin (in liver, heart, skin, large vessels, striated muscle, and lymphoid organs). ${ }^{36}{ }^{37}$ Both components are present in the chick eye in Descemet's membrane, the corneal stroma, and the elastic layer of Bruch's membrane. ${ }^{36}$ Colombatti et al, ${ }^{3}$ using immunofluorescence microscopy, noted 


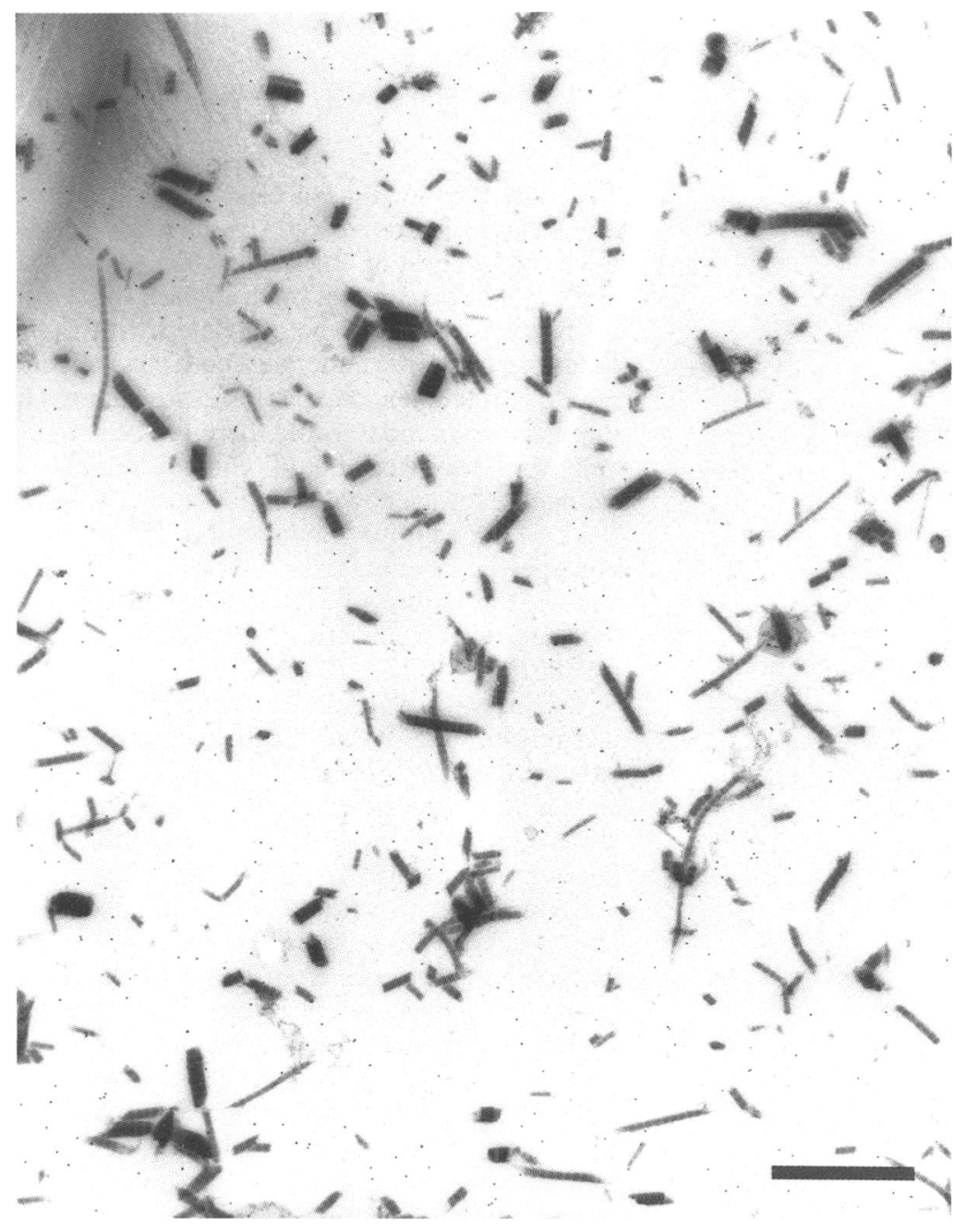

Figure 5 Labelling for fibronectin in stroma of normal iris appears to be randomly distributed in the electron lucent matrix and cannot be associated with any morphological structure (cryo, $\times 15500$, bar=l $\mu \mathrm{m}$ ). the presence of gp115 in the region of some, but not all basement membranes. Owing to the low resolution of the immunofluorescence technique, they were not able to establish if gpl15 is a true component of basement membranes. ${ }^{35}$

In the present study gpl15 was absent from basement membranes in both the normal and exfoliative iris but weakly labelled exfoliation material and regions of exfoliative iris stroma devoid of exfoliation aggregates. We regard these observations with some caution for, as with elastin, labelling could not be localised to specific structures in the iris stroma. The putative presence of gp115 in exfoliation material would represent the synthesis of a component novel to the iris vascular cell synthetic repertoire. This would lend further support to the suggestion of Streeten and co-workers ${ }^{1-316-18}$ that exfoliation material is derived from abnormal polymerisation of glycoproteins associated with the zonular elastic microfibrillar system in a type of elastosis. However, it should not be inferred that the synthesis of gpl15 is inevitably linked with the secretion of tropoelastin as cell culture experiments indicate that gp1 15 has different secretion kinetics to tropoelastin and a different fate after secretion. ${ }^{3 *}$

BASEMENT MEMBRANE COMPONENTS

\section{Entactin}

This is a sulphated basement membrane glyco- protein that may associate with the glycoprotein laminin in vivo. ${ }^{39}$ The distribution varies in different organs, sometimes being localised to the lamina densa of basement membranes, or to the basal surface of epithelial cells and on interstitial collagen fibrils. ${ }^{39}$ Owing to its frequent location at basal cell surfaces, entactin is suspected to be an attachment protein. ${ }^{39}$

We were unable to demonstrate the presence of entactin in either LR white or ultrathin frozen sections of both normal and exfoliative iris. We were therefore unable to confirm the results of Schlotzer-Schrehardt et al, who localised entactin to exfoliation deposits in eyelid skin, iris, and ciliary body, ${ }^{9}$ and on the lens surface. ${ }^{10}$ Our failure to localise entactin to exfoliation deposits cannot be explained by differences in fixation as one of our fixation regimes was virtually identical to that of Schlotzer-Schrehardt et al, ${ }^{9}$ (4\% PFA plus $0 \cdot 1 \%$ glutaraldehyde in cacodylate buffer for 2 hours). Differences in labelling could be attributed the use of different antibodies (that of Schlotzer-Schrehardt $e t a l^{9}$ was a personal gift whereas the one used in the present study was obtained commercially).

In our opinion the status of entactin in normal and exfoliative iris can only be suitably resolved by incorporation of a positive control - that is, a tissue in which the antigen is known to occur and which has been fixed, processed, and labelled in an identical manner. We are unaware of any normal ocular tissue in which entactin has been positively localised.

\section{Fibronectin}

This is a glycoprotein that exists in two forms; soluble (plasma) and insoluble (tissue). Since it binds to a number of biological macromolecules including fibrin, collagen, and heparin, and to a wide variety of cultured cells its main function is believed to be a cell matrix ligand..$^{39}$ Fibronectin is present in both loose connective tissue and basement membranes but there is some debate as to whether it should be considered an intrinsic constituent or simply arising from plasma. ${ }^{39} \mathrm{It}$ is abundant in developing tissue but is either absent or present in low amounts in mature tissues. ${ }^{+0}$

We did observe labelling for cellular fibronectin in the iris stroma on ultrathin frozen sections of both normal and exfoliative iris. There was no direct association of fibronectin antibodies with the stromal collagen fibrils and basement membranes were free of label. Exfoliative fibres did not exhibit labelling for fibronectin and we would therefore agree with Schlotzer-Schrehardt et al ${ }^{9}$ that fibronectin is not a component of exfoliation material.

\section{EXTRACELLULAR MATRIX COMPONENTS}

\section{Vitronectin}

This is a multifunctional glycoprotein that is present in plasma ( $\mathrm{S}$-protein) and the extracellular matrix of many tissues. ${ }^{+1+2}$ During adolescence, vitronectin together with amyloid $\mathrm{P}$ component, is localised with amorphous elastin at the periphery of dermal elastic fibres. ${ }^{+}$ It is absent from the skin of children before the 


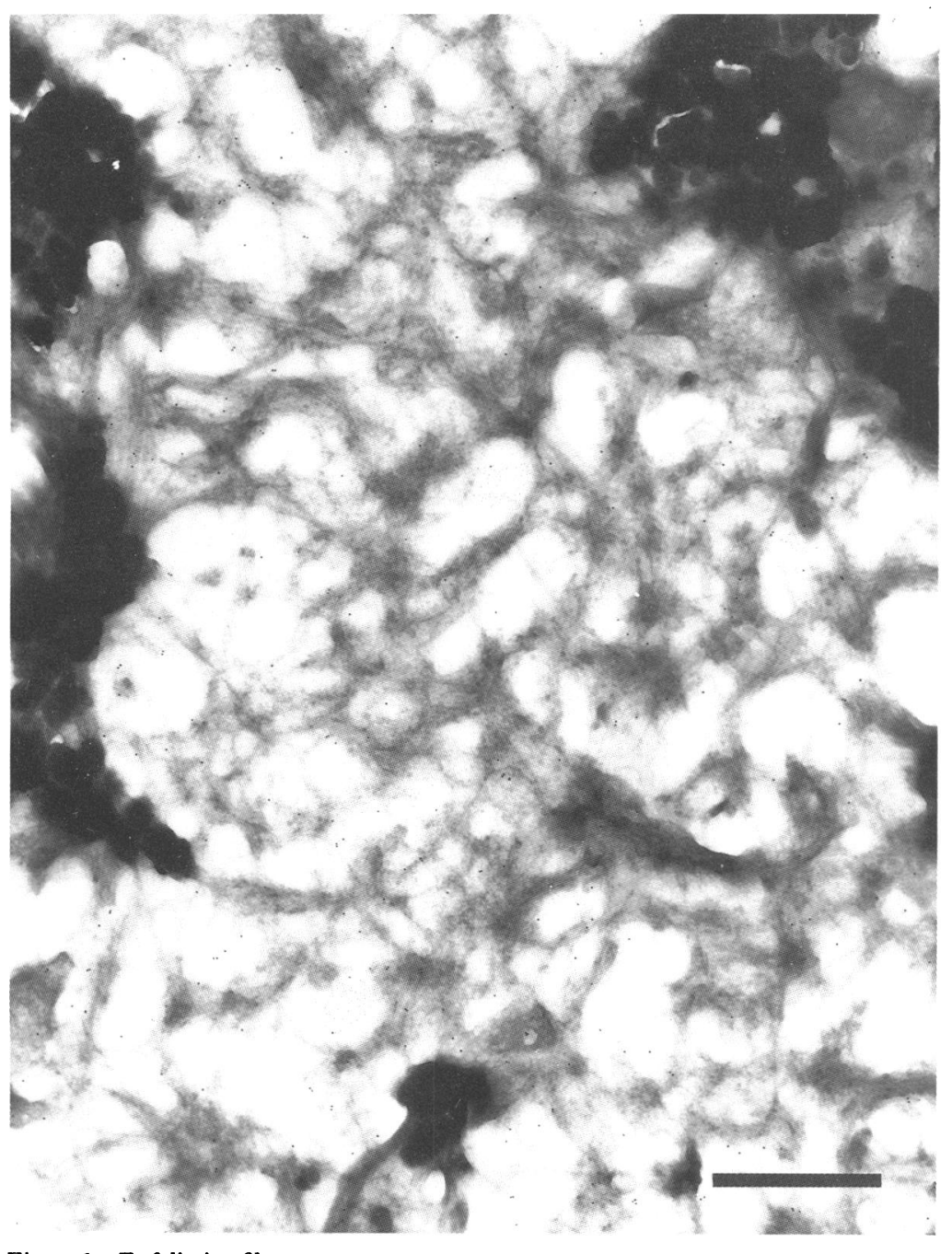

Figure 6 Exfoliation fibres are labelled with antibodies against elastin associated glycoprotein (cryo, $\times 18600$, bar $=1 \mu \mathrm{m})$. age of 6 and present in persons older than 13 years, ${ }^{44}$ where it is thought to serve as a molecular link between elastic fibres and the surrounding collagen/proteoglycan scaffold. ${ }^{43}$ Unlike fibronectin, vitronectin appears to be absent from basement membranes. ${ }^{45}$ Experimental evidence suggests that vitronectin provides a unique regulatory link between cell adhesion, humoral defence mechanisms, and cell invasion..$^{43}$

In spite of employing a number of fixation regimes and two tissue preparation techniques we were unable to localise vitronectin to any anatomical subcompartment in either normal or exfoliative iris. We were therefore unable to confirm the results of Schlotzer-Schrehardt et al, ${ }^{9}$ who localised vitronectin to exfoliation deposits in eyelid skin, iris, and ciliary body. This discrepancy cannot be explained by differences in fixation - one of our fixation regimes was virtually identical to theirs - nor by differences in antibody specificity, as the vitronectin antibody was supplied by the same company (Boehringer, Mannheim). As with entactin, the status of vitronectin in normal and exfoliative iris can only be suitably resolved by incorporation of a suitable positive control. We are unaware of any normal ocular tissue in which vitronectin has been positively localised.

It is not surprising that contradictions have arisen in the early stages of application of a novel technique which at this stage has the numerous pitfalls that we have already outlined. The logical extension from the data already accumulated is to determine which of the candidate cells are involved in the synthesis of these components. This can be achieved by the application of in situ hybridisation which will identify messenger RNA for the end product proteins.

1 Li Z-Y, Streeten BW, Wallace RN. Association of elastic with pseudoexfoliative material: an immunoelectron microscopic study. Curr Eye Res 1988; 7: 1163-72.

2 Streeten BW, Dark AJ, Wallace RN, Li ZY, Hoepner JA. Pseudoexfoliative fibrillopathy in the skin of patients with ocular pseudoexfoliation. Am $\mathcal{F}$ Ophthalmol 1990; 5: 490-9.

3 Streeten BW, Bookman L, Ritch R, Prince AM, Dark AJ. Pseudoexfoliative fibrillopathy in the conjunctiva. A relation to elastic fibers and elastosis. Ophthalmology 1987; 94: 1429-49.

4 Konstas AGP, Jay JL, Marshall GE, Lee WR. Prevalence, diagnostic features and response to trabeculectomy in exfoliation glaucoma. Ophthalmology 1993;100: 619-27.

5 Konstas AGP, Dimitracoulias N, Konstas PA. Exfoliation syndrome and open angle glaucoma. Klin Monatsbl syndrome and open angle

6 Lee WR. Ophthalmic histopathology. London: Springer Verlag,

7 Morrison JC, Green WR. Light microscopy of the exfoliation syndrome. Acta Ophthalmol (Suppl) 1988; 184: 5-27.

8 Spencer WH. Glaucoma. In: Ophthalmic pathology: an atlas and textbook. Philadelphia: Saunders, 1985: 480-547.

9 Schlotzer-Schrehardt U, Kuchle M, Dorfler S, Nauman GOH. Pseudoexfoliative material in eyelid-skin of pseudoexfoliation-suspect patients: a clinico-histopathological correlation. German f Ophthalmol 1993; 2: 51-60.

10 Schlotzer-Schrehardt U, Dorfler S, Naumann GOH. Immunohistochemical localization of basement membranes components in pseudoexfoliation material of the lens capsule. Curr Eye Res 1992; 4: 343-55.

11 Streeten BW, Li ZY, Wallace RN, Eagle RC, Keshgegian AA. Pseudoexfoliative fibrillopathy in visceral organs of a patient with pseudoexfoliation syndrome. Arch Ophthalmol 1992; 110: $1757-61$.

12 Konstas AGP, Marshall GE, Lee WR. Immunogold localisation of laminin in normal and exfoliative iris. Brf Ophthalmol 1990; 74: 450-7.

13 Konstas AGP, Marshall GE, Lee WR. Iris vasculopathy in exfoliation syndrome: an immunocytochemical study. Acta Ophthalmol 1991; 69: 472-85.

14 Newman GR, Jasani B, Williams ED. A simple postembedding system for the rapid demonstration of tissue antigens under the electron microscope. Histochem $\mathcal{F} 1983$; 15: $543-55$.

15 Grube D. Immunoreactivities of gastrin (G) cells II nonspecific binding of immunoglobulins to $\mathrm{G}$-cells by ionic interactions. Histochemisty 1980; 66: 149-67.

16 Streeten BW, Dark AJ, Barnes CW. Pseudoexfoliative material and oxytalan fibres. Exp Eye Res 1984 ; 38: 523-31.

$17 \mathrm{Li} \mathrm{ZY}$, Streeten BW, Yohai N. Amyloid P protein in pseudoexfoliative fibrillopathy. Curr Eye Res 1989; 8: 217-27.

18 Streeten BW, Gibson SA, Dark AJ. Pseudoexfoliative material contains an elastic microfibrillar-associated glycoprotein. Trans Am Ophthalmol Soc 1986; 84: 304-20.

19 Streeten BW, Gibson SA, Li ZY. Lectin binding to pseudoexfoliative material and the ocular zonules. Invest Ophthalmol Vis Sci 1986; 27: 1516-21.

20 Eagle RC, Font RL, Fine BS. The basement membrane exfoliation syndrome. Arch Ophthalmol 1979; 97: 510-5.

21 Konstas AGP, Marshall GE, Cameron SA, Lee WR. Morphology of iris vasculopathy in exfoliation glaucoma. Acta ology of iris vasculopathy
Ophthalmol 1993; 71: 751-9.

22 Konstas AG, Marshall GE, Lee WR. Immunocytochemical localisation of collagens (I-V) in the human iris. Graefes Arch Clin Exp Ophthalmol 1990; 39: 180-6.

23 Marshall GE, Konstas AG, Lee WR. Ultrastructural distribution of collagen types I-VI in aging human retinal vessels. Br F Ophthalmol 1990; 74: 228-32.

24 Marshall GE, Konstas AG, Lee WR. Immunogold fine structural localization of extracellular matrix components in aged human cornea. II. Collagen types V and VI. Graefes Arch Clin Exp Ophthalmol 1991; 229: 16+71.

25 Marshall GE, Konstas AGP, Lee WR. Immunogold ultrastructural localization of collagens in the aged human structural localization of collagens in the aged human
outflow system. Ophthalmology 1991; 98: 692-700.

26 Tuuthow system. Ophthalmologv 1991; 98: 692-700. Tokuyasu KT. Immunochemistry on ultrathin frozen sec-
tions. Histochem 7 1980; 12: 381-403.

27 Ringvold A. Exfoliation syndrome. Immunological aspects. Acta Ophthalmol 1988; 184: 35-43.

28 deBeer FC, Baltz ML, Holford S, Feinstein A, Pepys MB. Fibronectin and $\mathrm{C} 4$-binding protein are selectively bound by aggregated amyloid $P$ component. 7 Exp Med 1981: 154: 113449.

29 Dyck RF, Lockwood CM, Kershaw M, McHugh N, Duance VC, Balt\% ML, et al. Amyloid P-component is a constituent of normal human glomerular basement membrane. I E.ip
Med 1980; 152: 1162-74.

30 Brooks AMV, Gillies WE. The development of microvascular changes in the iris in pseudoexfoliation of the lens capsule. Ophthalmologv 1987; 94: 1090-7. 
31 Vannas A. Fluorescein angiography of the vessels of the iris in pseudoexfoliation of the lens capsule, capsular glaucoma and some other forms of glaucoma. Acta Ophthalmol Suppl 1969; 105: 9-75.

32 Bressan GM, Castellani I, Colombatti A, Volpin D. Isolation and characterization of a 115,000-dalton matrix-associated and characterization of a 115,000-dalton matrix-associated glycoprotein

33 Spring FA, Anstee DJ. Lectin-binding components of normal granulocytes and leukaemic myeloid cells. Biochem $\mathcal{F}$ 1983; 213: 661-70

34 Rosenstein Y, Park JK, Hahn WC, Rosen FS, Bierer BE, Burakoff SJ. CD43, a molecular defective in WiskottAldrich syndrome, binds ICAM-1. Nature 1991; 354: 233-4.

35 Colombatti A, Bressan GM, Castellani I, Volpin D. Glycoprotein 115 , a glycoprotein isolated from chick blood vessels, is widely distributed in connective tissue. 7 Cell Biol 1985; 100: $18-26$.

36 Colombatti A, Poletti A, Bressan GM, Carnone A, Volpi D. Widespread codistribution of glycoprotein gpl 15 and elastin in chick eye and other tissues. Coll Rel Res 1987; 7: 259-75.

37 Colombatti A, Poletti A, Carbone A, Volpin D, Bressan GM. Extracellular matrix of lymphoid tissues in the chick. $\mathcal{F}$ Histochem Cytochem 1989; 37: 757-63.
38 Colombatti A, Bonaldo P, Volpin D, Bressan GM. The elastin associated glycoprotein gpl15 synthesis and secretion by chick cells in culture. F Biol Chem 1988; 263: 17534-40.

39 Abrahamson DR. Recent studies on the structure and pathology of basement membranes. 7 Pathol 1986; 149: 257-78.

40 Liakka KA, Autio-Harmainen HI. Distribution of the extracellular matrix proteins tenascin, fibronectin, and vitronectin in fetal, infant and adult human spleens. $\mathcal{F}$ Histochem Cytochem 1992; 40: 1203-10.

41 Barnes D, Silnutzer J. Isolation of human serum spreading factor. F Biol Chem 1983; 258: 12548.

42 Hayman E, Pierschbacher M, Ohgren Y, Ruoslahti E. Serum spreading factor (vitronectin) is present at the cell surface and in tissues. Proc Natl Acad Sci USA 1983; 80: 4003.

43 Preissner KT. Structure and biological role of vitronectin. Ann Rev Cell Biol 1991; 7: 275-310.

44 Dahlback K, Lofberg H, Alumets J, Dahlback B. Immunohistochemical demonstration of age-related deposition of vitronectin (S-protein of complement) and terminal complevitronectin (S-protein of complement) and terminal complement complex on

45 Falk RJ, Podack E, Dalmasso AP, Jennette JC. Localization of $S$ Protein and its relationship to the membrane attack complex of complement in renal tissue. Am $\mathcal{F}$ Pathol 1987; 127: $182-90$. 